
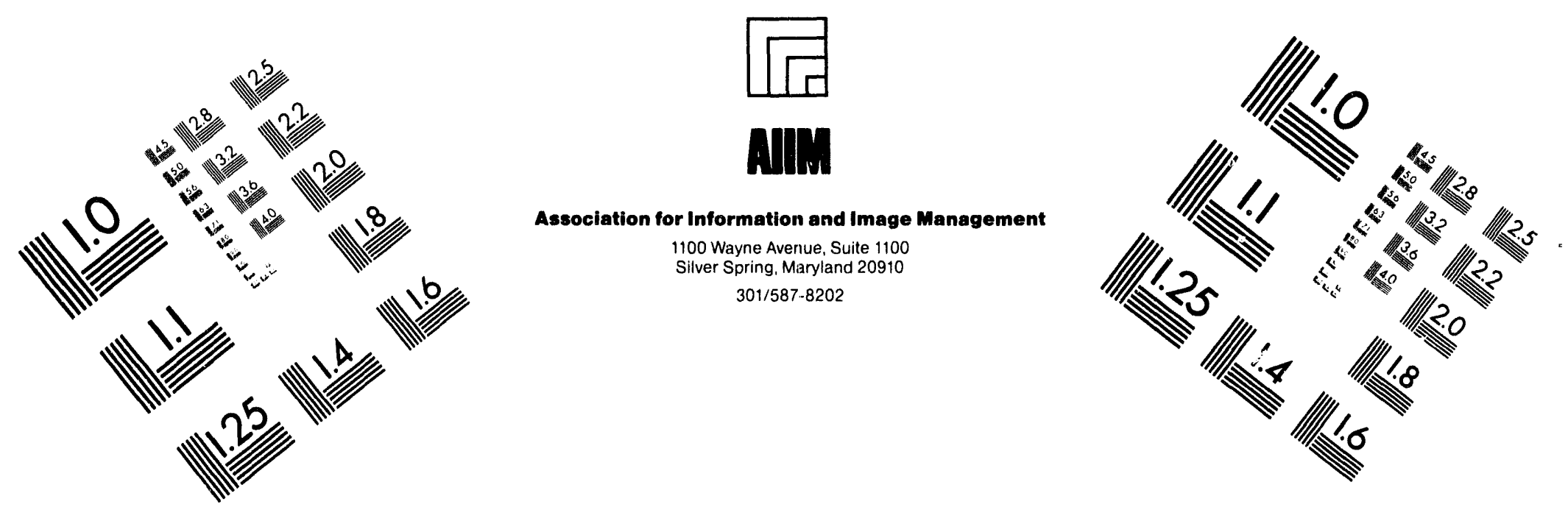

\title{
Centimeter
}

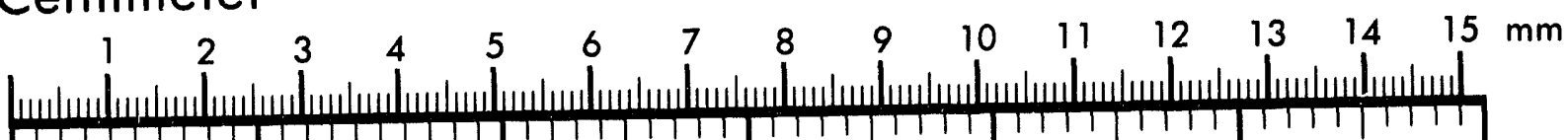

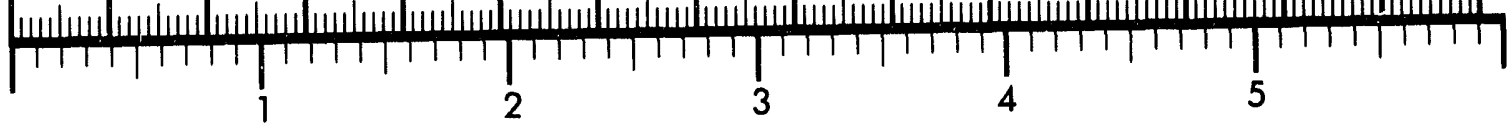
Inches
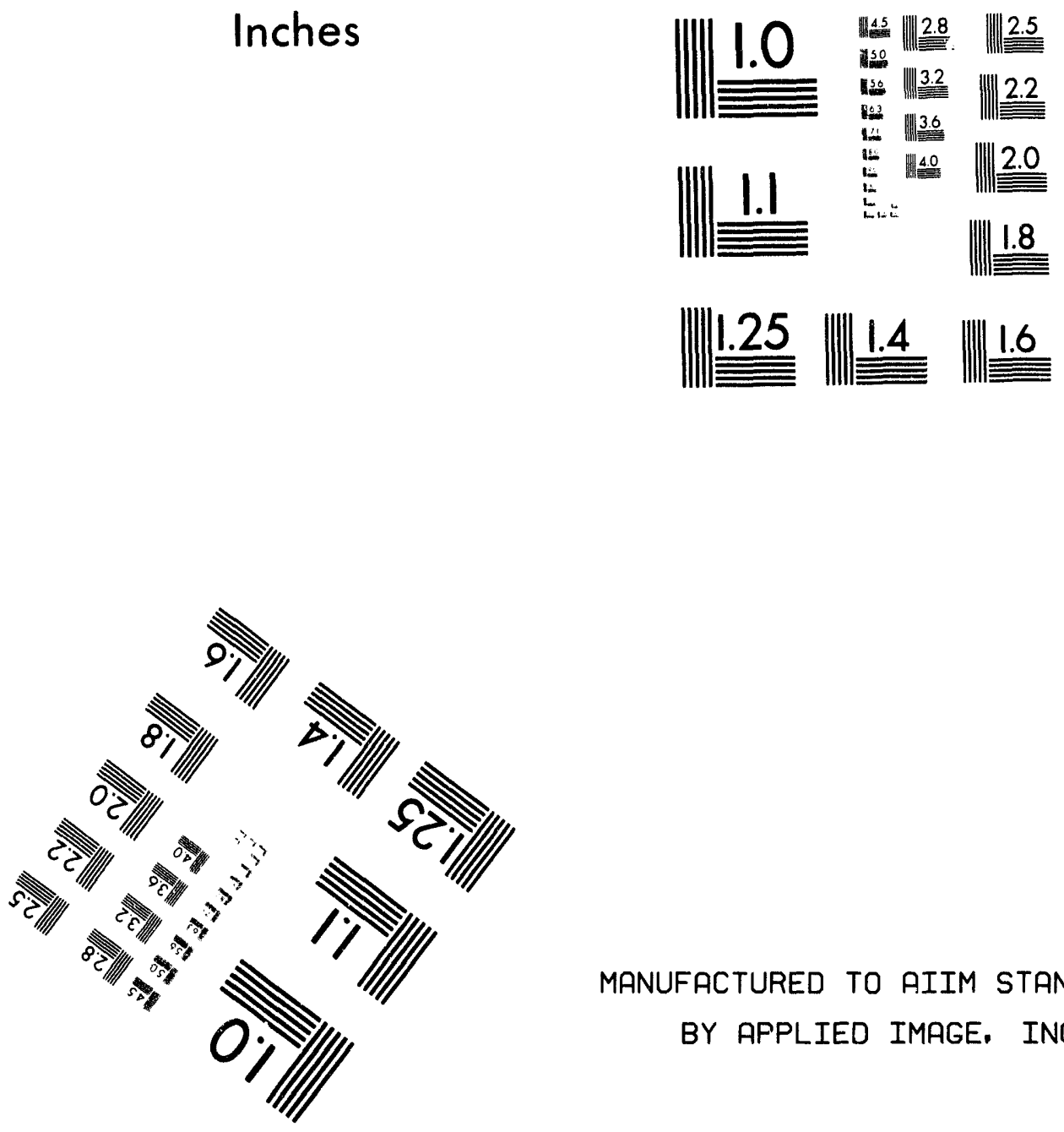

MANUFACTURED TO AIIM STANDARDS BY APPLIED IMAGE, INC.

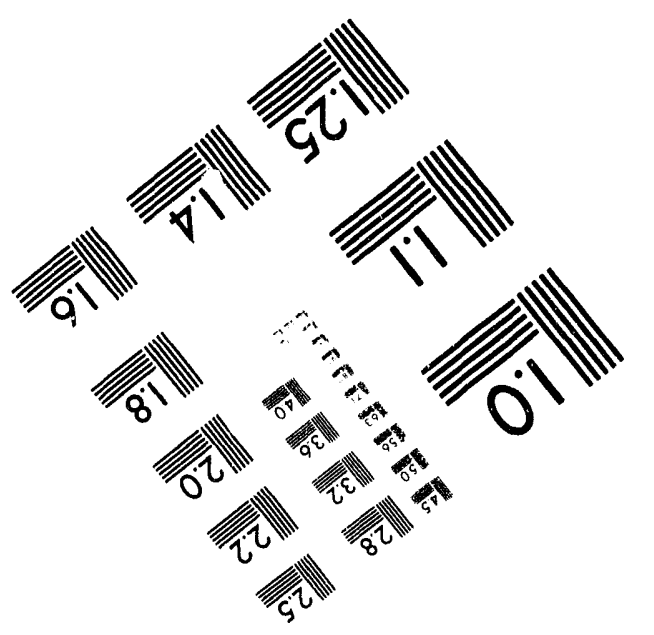



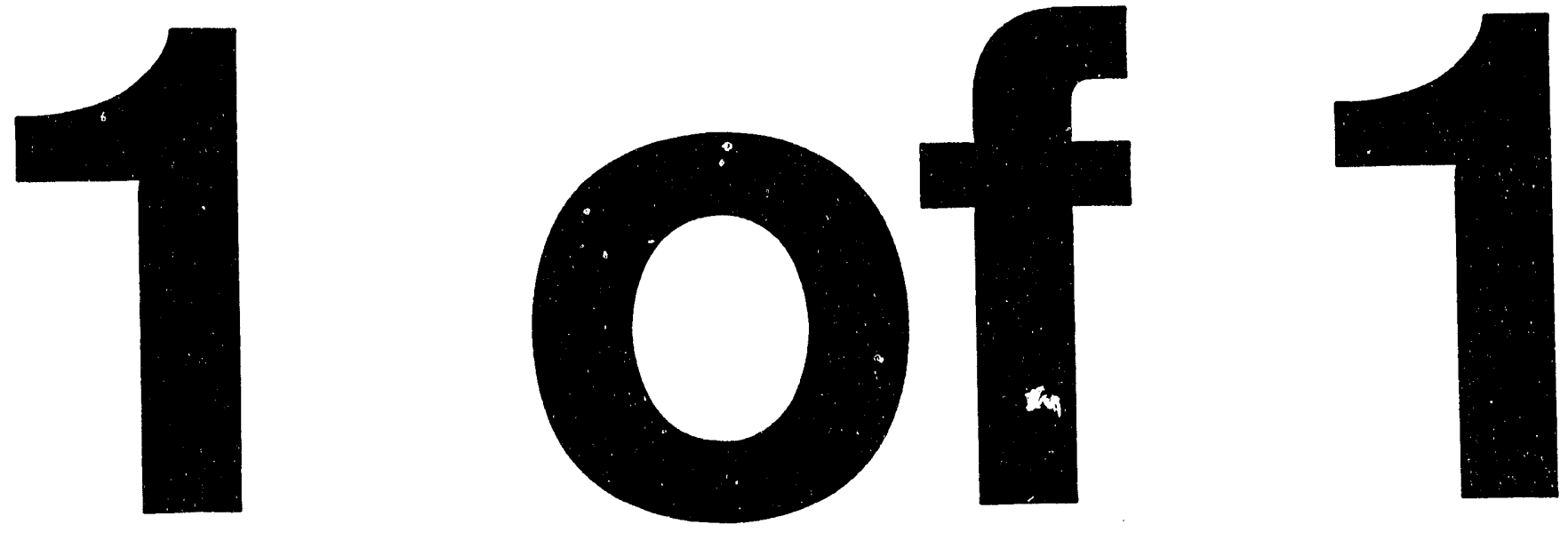
PNL-SA-24162

\section{HIGH PERFORMANCE COMPUTATIONAL CHEMISTRY; TOWARDS FULLY DISTRIBUTED PARALLEL ALGORITHMS}

\section{DISCLAIMER}

M. F. Guest et al

April 1994

\begin{abstract}
This report was prepared as an account of work sponsored by an agency of the United States Government. Neither the United States Government nor any agency thereof, nor any of their employees, makes any warranty, express or implied, or assumes any legal liability or responsibility for the accuracy, completeness, or usefulness of any information, apparatus, product, or process disclosed, or represents that its use would not infringe privately owned rights. Reference herein to any specific commercial product, process, or service by trade name, tradermark, manufacturer, or otherwise does not necessarily constitute or imply its endursement, recommendation, or favoring by the United States Government or any agency thereof. The views and opinions of authors expressed herein do not necessarily state or reflect those of the United States Government or any agency thereof.
\end{abstract}

Presented at the

1994 High Performance Computing Symposium

April 11-13, 1994

San Diego, California

Prepared for

the U.S. Department of Energy

under Contract DE-AC06-76RLO 1830

Pacific Northwest Laboratory

Richland, Washington 99352

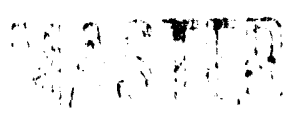




\title{
DUL-SA-24I b2
}

\author{
HIGH PERFORMANCE COMPUTATIONAL CHEMISTRY; \\ TOWARDS FULLY DISTRIBUTED PARALLEL ALGORITHMS
}

\author{
M. F. Guest, E. Apra, D. E. Bernholdt, H. A. Früchtl, R. J. Harrison, R. A. Kendall, \\ R. A. Kutteh, J, B. Nicholas, J. A. Nichols, M. S. Stave, and A. T. Wong \\ High Performance Computational Chemistry Group
}

and

R. J. Littlefield and J. Nieplocha

High Performance Computing Software Support

Molecular Science Research Center

Pacific Northwest Laboratory

PO Box 999, Mail Stop K1-90

Richland, WA. 99352

\section{ABSTRACT}

An account is given of work in progress within the High Performance Computational Chemistry Group (HPCC) at the Pacific Northwest Laboratory (PNL) to develop molecular modeling software applications for massively parallel processors (MPPs). A discussion of the issues in deveioping scalable parallel algorithms is presented, with a particular focus on the distribution, as opposed to the replication, of key data structures. Replication of large data structures limits the maximum calculation size by imposing a low ratio of processors to memory. Only applications that distribute both data and computation across processors are truly scalable. The use of shared data structures, which may be independently accessed by each process even in a distributed-memory environment, greatly simplifies development and provides a significant performance enhancement. In describing tools to support this programming paradigm, an outline is given of the implementation and performance of a highly efficient and scalable algorithm to perform quadratically convergent, self-consistent field calculations on molecular systems. A brief account is given of the development of corresponding MPP capabilities in the areas of periodic Hartree Fock, Möller-Plesset perturbation theory (MP2), density functional theory, and molecular dynamics.
Performance figures are presented using both the Intel Touchstone Delta and Kendall Square Research KSR2 supercomputers.

\section{INTRODUCTION}

The mission of the HPCC group at PNL is to develop molecular modeling software applications that provide 10-100 times more computing capability than has been available with conventional supercomputers. While increases in raw computing power alone. will greatly expand the range of problems that can be treated by theoretical chemistry methods, a significant investment in new algorithms is needed to fully exploit the potential of MPPs. Merely porting presently available software to these parallel computers does not provide the efficiency required to exploit their full potential. Most existing parallel applications show a significant deterioration in performance as greater numbers of processors are used. In some cases, the efficiency is so poor that the use of additional processors derreases, rather than increases, the performance. Thus, new algorithms must be developed that exhibit parallel scalability (i.e., show a near linear increase in performance with the number of processors). Although perfect scalability is very difficult to achieve, we have demonstrated our 
ability to approach this level of performance with our new self-consistent field (SCF) codes (see below).

Another important consideration in using MPPs is how data are stored. So-called replicated-data schemes require that a copy of each data item in the program be stored on each processor, so that the size of the problem that can be handled is limited by the memory of a single processor. In distributed-data applications each processor holds only a part of the total data; in such cases, the problem size is limited only by the total memory of the machine, allowing much larger problems to be treated. Our efforts focus on distributed-data applications. They span virtually the whole range of computational chemistry methods, with significant efforts in Hartree-Fock and correlated techniques, multiconfiguration SCF methods, density functional theory (DFT), semi-empirical methods, and classical Monte Carlo (MC) and molecular dynamics (MD).

In addition to the creation of new algorithms for computational chemistry on parallel processors, we are also creating the high-level data and control structures needed to make parallel programs easier to write, maintain, and extend. We have developed portable tools for memory allocation, message passing, distributed global arrays, performance analysis, decomposition strategies, prototyping of new kernels, refinement of performance models, code architecture determination, and teraflops projections. Our applications feature major design improvements over traditional codes and are modular at all levels. In concert with the PNL Extensible Computational Chemistry Environment effort, others associated with our project are also creating graphical user interfaces and molecular visualization modules, and are linking all of our computational codes to an objectoriented database. This allows for easy and accurate input, visualization, storage, analysis, and retrieval of molecular geometries and properties.

\section{SOFTWARE DEVELOPMENT TOOLS}

No emerging standards for parallel programming languages (notably just High Performance FORTRAN
[HPF-1]) provide extensive support for MIMD programming (Kendall et al. 1994). The only truly portable multiple instruction multiple data (MIMD) programming model is message passing, for which a standard interface has been recently proposed (MPI Forum, 1993). It is, however, very hard to develop applications with fully distributed data structures using the message-passing model (Colvin et al. 1993; Furlani and King 1993). What is needed is support for one-sided access to data structures (here limited to one- and two-dimensional arrays) in the spirit of shared memory. With some effort this can be done portably (Nieplocha et al. 1994). In return for this investment, we gain a much easier programming environment, speeding code development and improving extensibility and maintainability.

We also gain a significant performance enhancement from increased asynchrony of execution of processes (Arango et al. 1990). Message passing forces processes to cooperate (e.g., by responding to requests for a particular datum). Inevitably, this involves waiting for a collaborating process to reach the same point in the algorithm, which is only partially reduced by the use of complex buffering and asynchronous communication strategies. With a one-sided communication mechanism, where each process can access what it needs without explicit participation of another process, all processes can operate independently. This approach eliminates unnecessary synchronization and naturally leads to interleaving of computation and communication.

Most programs contain multiple algorithms, some of which may naturally be task-parallel (e.g., Fock matrix construction), and others that may be efficiently and compactly expressed as data-parallel operations (e.g., evaluating the trace of a matrix product). Both types of parallelism must be efficiently supported.

Consideration of the requirements of the SCF algorithm discussed below, and also the parallel COLUMBUS configuration interaction program (Schuler et al. 1993), MP2 theory (Bernholdt and Harrison 1994) and parallel Coupled-Cluster 
approach is to distribute all large data structures of size $O\left(N^{2}\right)$ and use a moderately course-grained, strip-mined approach to the Fock matrix construction. In addressing the latter, the SCF theory has been reformulated so as to avoid the diagonalization step altogether (Shepard 1993; Pollard and Friesner 1993). The sequential $O\left(N^{3}\right)$ diagonalization step is replaced by a quadratically convergent NewtonRaphson minimization, using data-parallel operations and matrix multiplication.

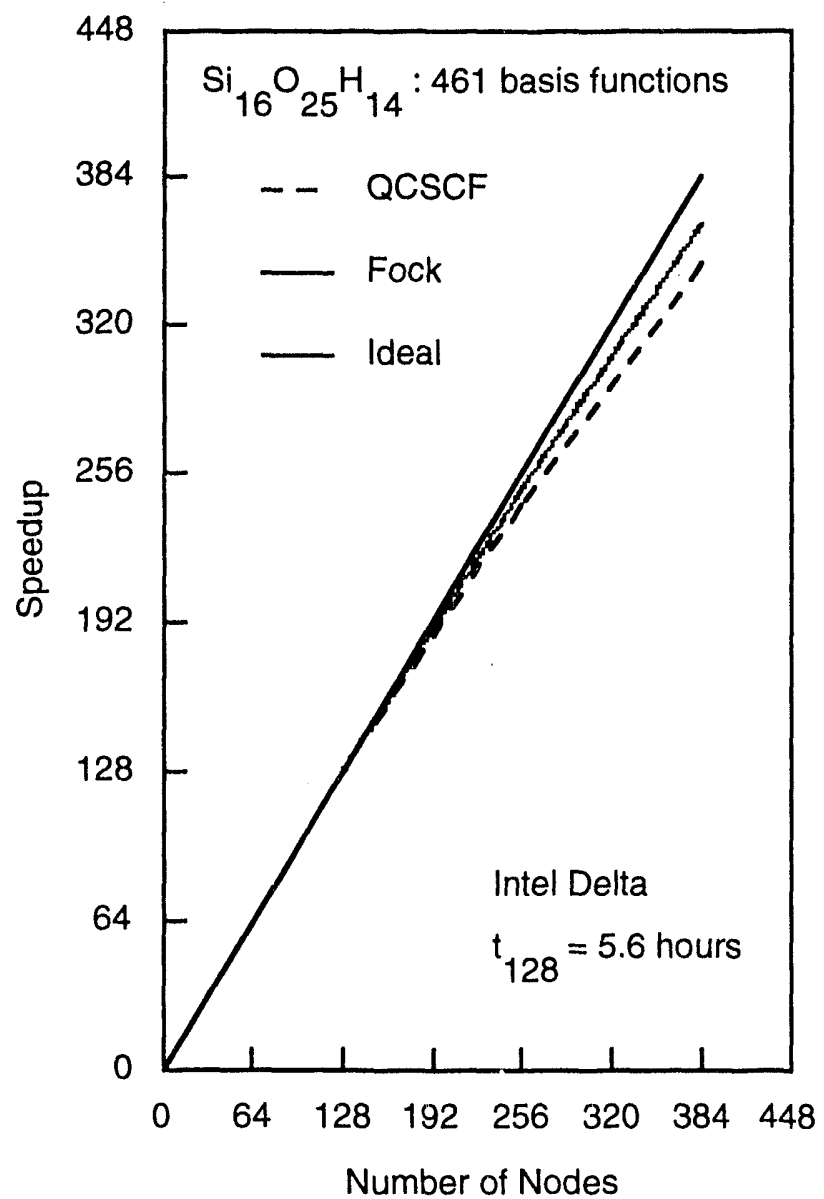

Figure 1. Quadratically Convergent SCF (QCSCF) performance on the Intel Touchstone Delta.

Figures (1-2) show the speedup for the distributed Fock matrix construction and the complete QCSCF algorithm for the 461 basis function calculation of disilasesquioxane on both the Intel Touchstone Delta (Figure 1) and the KSR-2 (Figure 2). When the number of processors is of order $\mathrm{Natom}^{2} / 2$, a degradation in the speedup for Fock matrix construction is observed due to load-balancing. The overall QCSCF speedup for small molecular calculations is also affected by the finer distribution of the global data structures as the number of nodes increases. In the disilasesquioxane case, neither effect is evident; the scalability of the QCSCF algorithm using distributed data has been demonstrated up to 512 nodes with systems of $N \sim 700$.

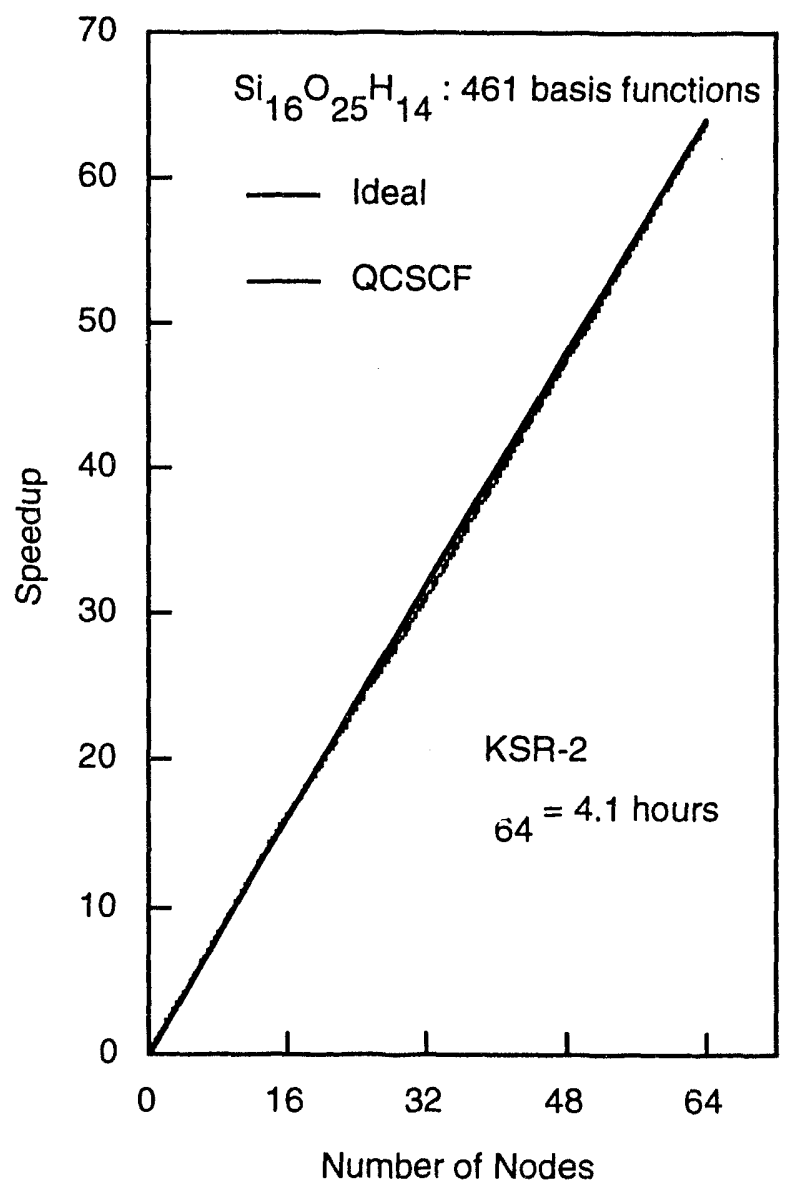

Figure 2. QCSCF performance on the KSR-2.

\section{Periodic Hartree Fock; Crystal 92}

The periodic Hartree Fock code, Crystal (Dovesi et al. 1992; Harrison and Saunders 1994), has been parallelized initially using message passing (with theoretical chemistry group message passing (TCGMSG) (Harrison 1991)) and a replicated data approach. It has recently been interfaced with a parallel virtual machine version 3 (PVM3) harness in which it was necessary to code FORTRAN routines 
electron correlation. The simplest such method is MP2 Theory, which has proven useful in a wide range of chemical problems including those for which the Hartree-Fock method is inadequate (Szabo and Ostlund 1989). A fully-distributed MP2 code is currently at the prototype stage, and for some relatively small systems, shows good scalability to 256 processors on the Intel Delta.

The large, spatially-extended molecular systems we are initially targeting are in general characterized by locality of interactions; distant parts of the system have very little effect on each other. We take advantage of this with an orbital invariant MP2 formulation utilizing the original atomic orbital (AO) basis set rather than the more delocalized molecular orbitals (MOs) of the traditional approach (Pulay and Saebo 1986). The local nature of the interactions manifests itself as block sparsity in the tensors describing them in the MP2 method. Using the existing parallel MP2 code, we will be able to experiment on systems large enough to display this sparsity and determine how best to take advantage of it while retaining, or hopefully improving, the scalability of the code.

The MP2 involves $\mathrm{O}\left(\mathrm{N}^{5}\right)$ computational work and $\mathrm{O}\left(\mathrm{N}^{4}\right)$ data, as compared with $\mathrm{O}\left(\mathrm{N}^{4}\right)$ and $\mathrm{O}\left(\mathrm{N}^{2}\right)$, respectively, for the SCF. Figure 4 illustrates the speedup of an MP2 augmented pVDZ calculation on $\mathrm{C}_{4} \mathrm{H}_{10}$ performed on the Intel Touchstone Delta. The two dominant computational steps are the construction of the exchange operators and the determination of the excitation amplitudes. Exceptional speedup is shown for the first step, while degradation is shown for the second step due to the smallness of system size and relatively high ratio of communication-to-computation time.

We believe that once sparsity is incorporated in the parallel code, we will be able to treat systems on the order of 1000-3000 basis functions, or 100-300 atoms, nearly an order of magnitude larger than the largest reported MP2 calculations to date. The much larger amount of data involved in an MP2 calculation will limit the size of calculation unless scalable I/O facilities are available to make disk storage of some of the data practical.

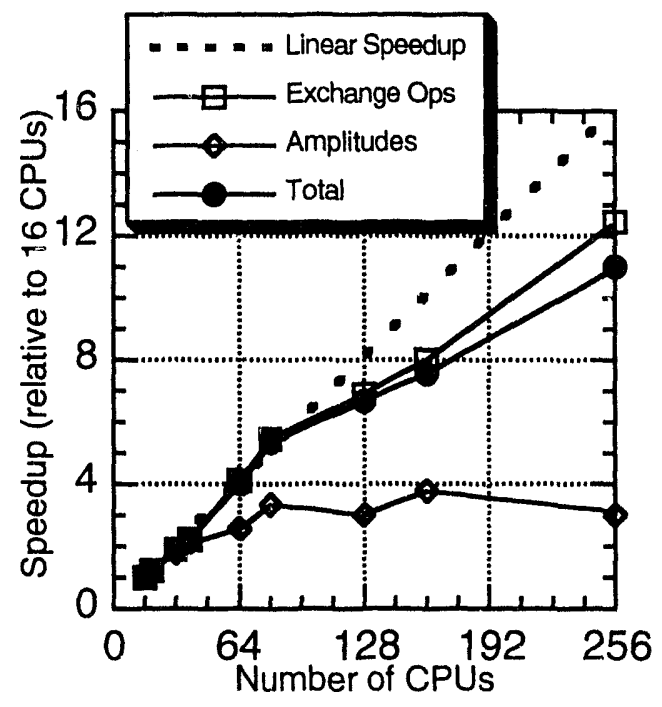

Figure 4. Speedup of an MP2 augmented pVDZ calculation on $\mathrm{C}_{4} \mathrm{H}_{10}$ performed on the Intel Touchstone Delta

\section{Density Functional Theory}

We are developing a parallel implementation of the Hohenberg-Kohn-Sham formalism (Hohenberg and Kohn 1964; Kohn and Sham 1965) of density functional theory (DFT). DFT is able to give results similar to those from correlated ab initio methods at substantially less cost. DFT assumes a charge density and successively better approximates the Hamiltonian (the exchange correlation functional). This method contrasts sharply with traditional ab initio molecular orbital methods, which assume an exact Hamiltonian and successively better approximates the wavefunction (Wimmer 1991). In our Gaussian basis DFT method the Hamiltonian is broken down into the same basic one-electron and twoelectron components. The two-electron component is further broken down into a Coulomb term and an exchange-correlation term. The electron density and the exchange-correlation functional can also be expanded in terms of Gaussian basis sets. 


\section{Molecular Dynamics}

We are developing a full-featured, general-purpose code for MD simulations on MPPs, designed to treat virtually all chemical systems, with the same functionality as many commercial programs.

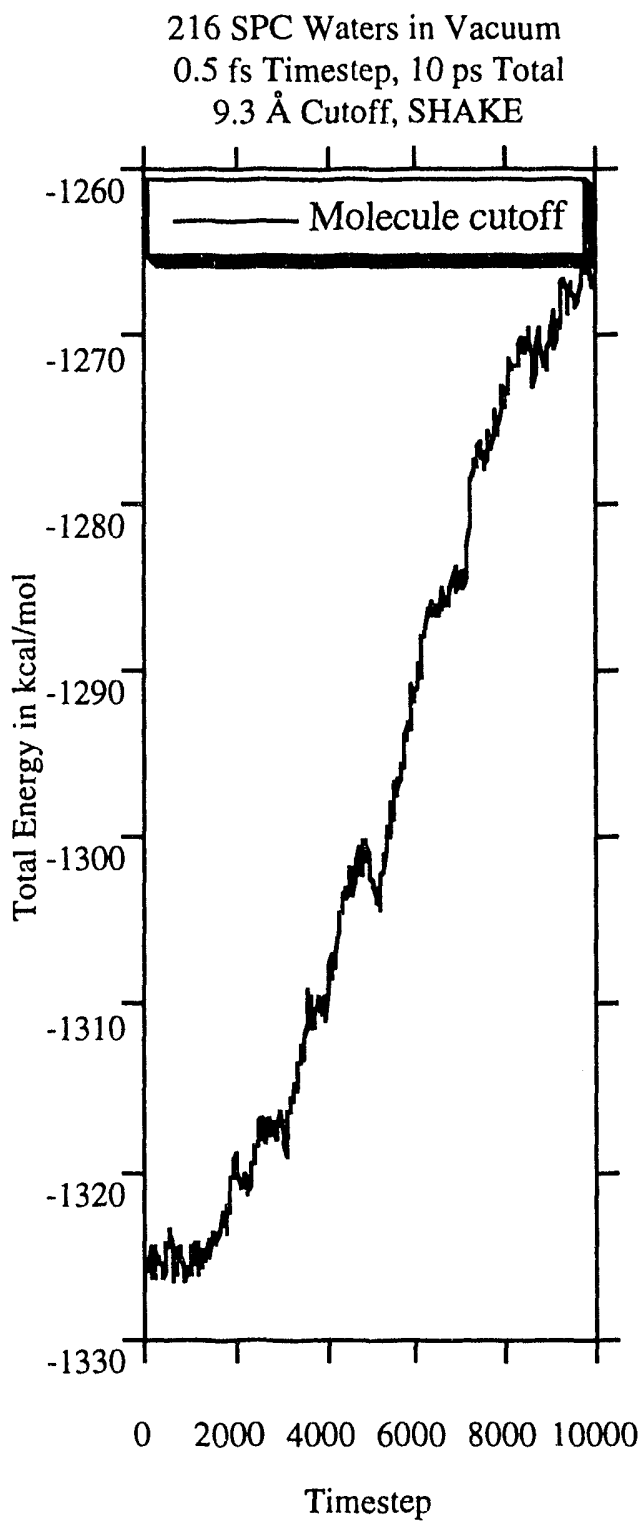

Figure 6. Inaccuracies when using a cutoff to evaluate Coulomb interactions.

The code features an extensive and easily modifiable force field, efficient treatment of long range interactions via Ewald summations, and fast multipole methods, a variety of statistical mechanical ensembles, energy minimizations, and normal mode calculations. The code runs on a wide range of MPPs and gives large performance gains over scalar and vector computers. For example, on 516 processors of the Intel Delta, our code requires less than a tenth of a second to perform a step of MD on 7776 atoms. We have performed some of the largest MD simulations to date studying diffusion in zeolites to better understand separation processes in these materials. These nanosecond simulations involved $\approx 10,000$ atoms and required $\approx 100$ hours on the Intel Delta. We are developing a distributed data version of this code that will extend the size of systems that can be studied to beyond a million atoms.

The calculation of the forces is by far the most time consuming step in an MD simulation. Using a cutoff to overcome this difficulty leads to inaccuracies in the case of Coulomb interactions as shown in Figure 6. The Fast Multipole Method (FMM) (Greengard 1987) (spherical harmonics) and its Cartesian counterpart, the Cell Multipole Method (CMM) (Ding et al. 1992), reduce the computation of Coulomb type sums from order $\mathrm{N}^{2}$ (direct method) to order $\mathrm{N}$. These methods use a divide and conquer strategy, multipole and Taylor expansions of potentials, and shifting of the multipole and Taylor coefficients between successive levels to achieve their speed. The FMM and CMM approaches use a tree structure where a cell at level (l) is divided into eight children cells at level $(1+1)$, each of which is divided in turn into eight children cells at level $(1+2)$, and so on as illustrated below in Figure 7.

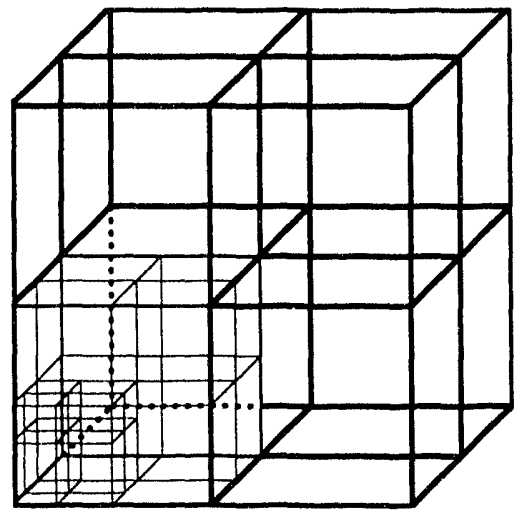

Figure 7. Tree structure used in FMM and CMM MD calculations. 
Ding, H.-Q.; N. Karasaw'a; and W. A. Goddard III. 1992. "Atomic level simulations on a million particles; The cell multipole method for Coulomb and London nonbond interactions." J. Chem. Phys., 97: 4309-4315.

Dovesi R., V. R. Saunders and C. Roetti, 1992. "CRYSTAL92 user's manual", University of Torino, Torino. Harrison N. M. and Saunders V. R., 1994. CCP Quarterly Newsletter, S.E.R.C Daresbury Laboratory, Daresbury.

Feyereisen, M.; and R. A. Kendall. 1993. "An efficient implementation of the direct-SCF algorithm on parallel computer architectures." Theoretica Chimica Acta, 84: 289-299.

Furlani, T. R. ; and H. F. King. 1993. "Implementation of a parallel direct SCF algorithm on a distributed memory computer." J. of Comp. Chem. (in press).

Greengard, L. 1987. "The rapid evaluation of potential fields in particle systems." MIT Press, Cambridge.

Harrison, R. 1991. Int. J. Quant. Chem., 40: 847. TCGMSG is available by anonymous ftp from ftp.tcg.anl.gov or by sending electronic mail to rj_harrison@pnl.gov.

Hohenberg, P.; and W. Kohn. 1964. "Inhomogeneous Electron Gas" Phys. Rev. B., 136: 864-871.

Kendall, R. A. ; R. J. Harrison; R. J. Littlefield; and M. F. Guest. 1994. "High Performance Computing and Computational Chemistry: a Review of Methods and Machines." To appear in Reviews in Computational Chemistry, K. B. Lipkowitz and D. B. Boyd, eds. VCH Publishers, Inc., New York.

Kohn, W.; and L. J. Sham. 1965. "Self-consistent equations including exchange and correlation effects" Phys. Rev. A., 140: 1133-1138.

"MPI: A Message Passing Interface", The MPI Forum, 1993, Proceedings of Supercomputing '93. IEEE computer Society Press, Los Alamitos, California 878883.

Nieplocha, J.; R. J. Harrison; and R. J. Littlefield. 1994. "Global arrays; A portable shared memory programming model for distributed memory computers." to appear in Supercomputing '94, IEEE Computer Society Press.

Perdew, J. P. 1986. "Density-functional approximation for the correlation energy of the inhomogeneous electron gas." Phys. Rev. B., 33(12): 8822-8824.

Pollard, W.T.; and R. A. Friesner. 1993. "Efficient Fock Matrix diagonalization by a Krylov-space method." J. Chem. Phys., 99: 6742-6750.

Pulay, P.; and S. Saebo. 1986. "Orbital-invariant formulation of a second-order gradient evaluation in Möller-Plesset Perturbation theory." Theoretica Chimica Acta, 69: 357-368.

Rendell A. P.; M. F. Guest; and R. A. Kendall. 1993. "Distributed Data Parallel Coupled-Cluster Algorithm: Application to the 2-Hydoxypyridine / 2-Pyridone Tautomerism." J. Comp. Chem., 14: 14291439.

Schuler, M.; T. Kovar; H. Lischka; R. Shepard; and R. J. Harrison. 1993. "A parallel implementation of the COLUMBUS multireference configuration interaction program." Theoretica Chimica Acta, 84: 489-509.

Shepard, R. 1993. "Elimination of the diagonalization bottleneck in parallel Direct-SCF methods." Theoretica Chimica Acta, 84: 343-351

Szabo, A; and N. S. Ostlund. 1989. "Modern Quantum Chemistry." McGraw-Hill, N. Y.

Wimmer, E. 1991. "Density functional theory for solids, surfaces, and molecules: from energy bands to molecular bonds." In Density functional methods in 

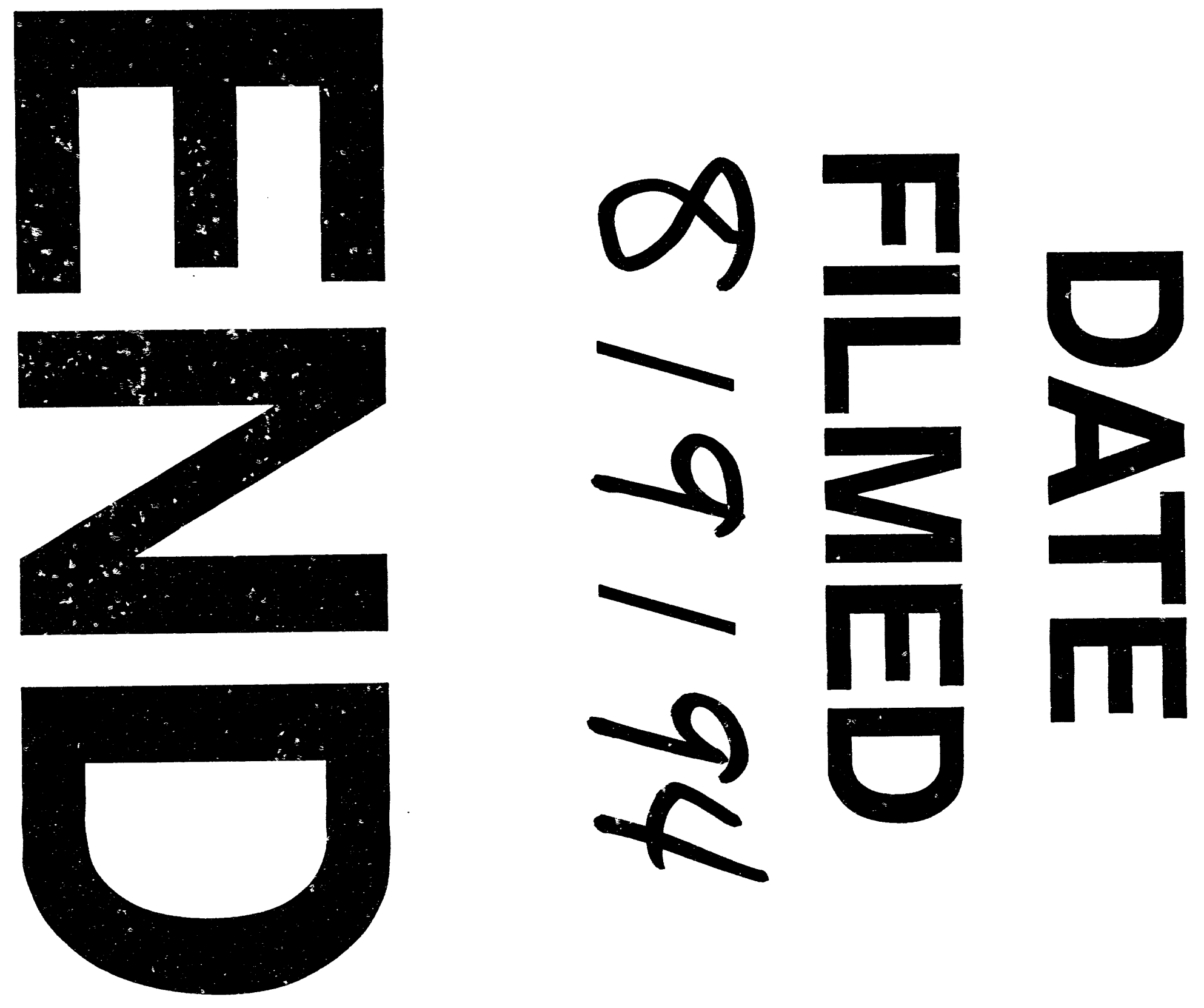
